\title{
Análise do eixo de rotação da terra no Japão considerando a ocorrência de terremoto ocorrido em março de 2011
}

Objetivou-se analisar o eixo de rotação da terra no período superior a um ano, considerando a ocorrência de um fenômeno natural, como o terremoto do Japão ocorrido em março de 2011, para saber se o mesmo contribuiu para alterar o eixo de rotação da Terra e quais as variações sofridas durante o período de estudo. Foram coletados os dados na página oficial do IERS (International Earth Rotation and Reference Systems Service) os dados de orientação da Terra, que são encontrados nos Boletins B, enumerados e coletados no intervalo do boletim B 278 até boletim B 285, que representam o período de setembro de 2010 a setembro de 2011. Com o uso do Excel 2016, tabelou-se e organizaram-se os dados, os quais se referem ao movimento do polo de $\mathrm{x}$ e y na unidade de milésimo de segundo (mas). Feito a organização, houve a criação do gráfico no próprio software. As figuras expostas demonstram que as oscilações obedeceram ao padrão cíclico do Movimento de Chandler, de modo que é possível inferir que, apesar da magnitude do evento, não houve interferência sobre o movimento analisado.

Palavras-chave: Astronomia de Posição; Movimento do Polo; Movimento de Chandler

\section{Analysis of the axis of rotation of the earth in Japan considering the occurrence of an earthquake that occurred in march 2011}

The objective was to analyze the axis of rotation of the earth over a period of more than a year, considering the occurrence of a natural phenomenon, such as the earthquake in Japan in March 2011, to find out if it contributed to alter the axis of rotation of the Earth and what the variations suffered during the study period. Data were collected on the official page of the IERS (International Earth Rotation and Reference Systems Service), Earth orientation data, which are found in Bulletins B, listed and collected in the interval from bulletin B 278 to bulletin B 285, which represent the period from September 2010 to September 2011. Using Excel 2016, the data were tabulated and organized, which refer to the $\mathrm{x}$ and y pole movement in the millisecond (but) unit. After the organization, the graphic was created in the software itself. The exposed figures show that the oscillations obeyed the cyclical pattern of the Chandler Movement, so that it is possible to infer that, despite the magnitude of the event, there was no interference on the analyzed movement.

Keywords: Position Astronomy; Pole Movement; Chandler Movement

Topic: Tecnologia, Modelagem e Geoprocessamento

Reviewed anonymously in the process of blind peer.
Received: 20/04/2021

Approved: 30/05/2021
Breno Eduardo dos Santos Alves (ic)

Universidade Federal Rural da Amazônia, Brasil

http://lattes.cnpq.br/7871130001431912

http://orcid.org/0000-0003-4500-821X

brenoedu33@gmail.com

Alexandre Santos Fernandes Filho (D)

Universidade Federal Rural da Amazônia, Brasil

http://lattes.cnpq.br/0578757011156726

http://orcid.org/0000-0002-1684-3788

alexandresff25@gmail.com

Hellem Cristina Teixeira Rodrigues (iD

Universidade Federal Rural da Amazônia, Brasil

http://lattes.cnpq.br/3666066794430752

http://orcid.org/0000-0003-2671-8742

hellem.cristinat@gmail.com

\author{
Marcus Vinicius Zamorim da Costa \\ Universidade Federal Rural da Amazônia, Brasil \\ http://lattes.cnpq.br/8014371678876754 \\ http://orcid.org/0000-0002-9090-9669 \\ marcusvzamorim@gmail.com \\ Rayssa Soares da Silva (it \\ Universidade Federal Rural da Amazônia, Brasil \\ http://lattes.cnpq.br/3334094744060074 \\ http://orcid.org/0000-0003-4626-6384 \\ rayssacereja@gmail.com \\ Francimary da Silva Carneiro \\ Universidade Federal Rural da Amazônia, Brasil \\ http://lattes.cnpq.br/8657235544233319 \\ http://orcid.org/0000-0002-1693-8779 \\ francimarycarneiro@gmail.com
}

Referencing this:

DOI: 10.6008/CBPC2237-9290.2021.002.0014

ALVES, B. E. S.; FERNANDES FILHO, A. S.; RODRIGUES, H. C. T.; COSTA M. V. Z.; SILVA, R. S.; CARNEIRO, F. S.. Análise do eixo de rotação da terra no Japão considerando a ocorrência de terremoto ocorrido em março de 2011. Natural Resources, v.11, n.2, p.133-137, 2021. DOI: http://doi.org/10.6008/CBPC2237-9290.2021.002.0014 


\section{INTRODUÇÃO}

Do ponto de vista da astronomia moderna, a Terra é uma plataforma bastante irregular para a observação do céu. A rotação da Terra não é uniforme, seu eixo de rotação não é fixo no espaço e mesmo a forma do planeta e as posições relativas de pontos sobre sua superfície não são fixas. Como resultado, as coordenadas de um objeto no céu não são rigorosamente constantes. Se mudar a direção do eixo de rotação, por exemplo, mudam os valores da ascensão reta $\alpha$ e da declinação $\delta$ de todos os objetos na esfera celeste. Dependendo da precisão com que se deseja medir a posição das estrelas, faz-se necessária a correção para estes efeitos (SANTIAGO, 2019).

O eixo de rotação da Terra varia com respeito à figura da Terra, principalmente pelas propriedades elásticas e interação com a atmosfera, ocasionando o movimento de precessão, mutação e movimento do polo (ALVES et al., 2008). Poucos trabalhos abordam essa vertente se comparados a outros temas, no entanto, analisar a variação dos movimentos do eixo da terra é primordial para o estudo da geodesia moderna, que busca a medição e localização precisa na terra, assim como outras áreas do conhecimento.

Com isso, este trabalho objetivou analisar o eixo de rotação da terra no período superior a um ano, considerando a ocorrência de um fenômeno natural, como é o caso de terremoto do Japão ocorrido em março de 2011. Assim, analisar o fenômeno contribuiu para alterar o eixo de rotação da Terra e quais as variações sofridas durante o período de estudo.

\section{METODOLOGIA}

A área de estudo é o Japão, país que sofreu com o $7^{\circ}$ mais forte terremoto da história, de acordo com a BBC News (2011). O fenômeno, de magnitude 8.8, ocorreu em uma sexta-feira, dia 11 de março de 2011. Segundo a USGS (United States Geological Survey), o mais forte terremoto da história ocorreu em 22 de maio de 1960, em Valdívia (Chile), magnitude 9.5.

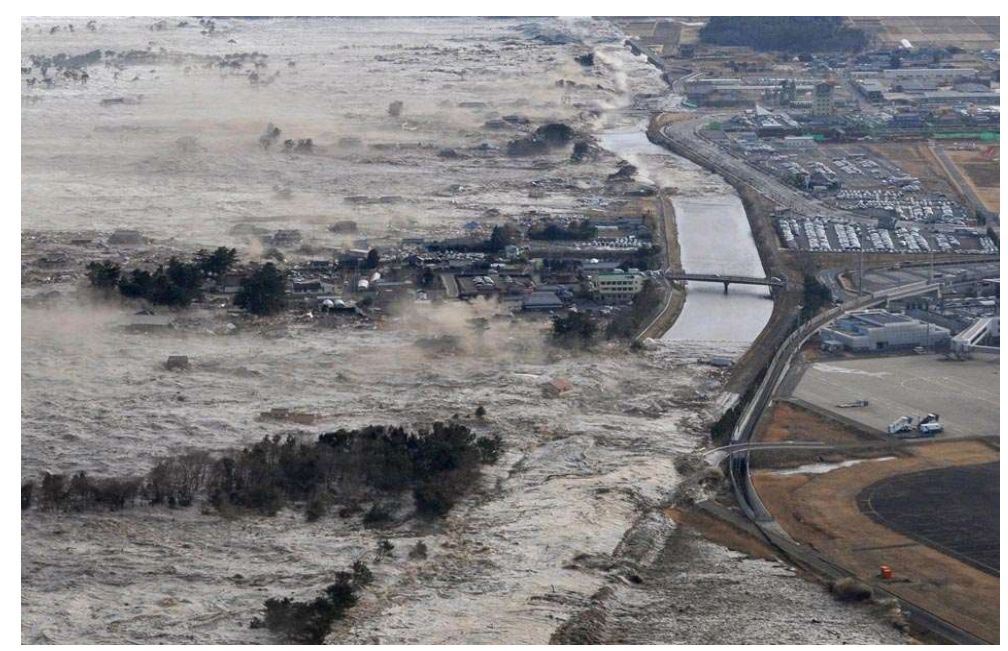

Figura 1: Iwanuma, norte do Japão. Fonte: Kyodo News/AP

O terremoto atingiu o Japão por volta de $15 \mathrm{~h}$ (hora local) e seu epicentro foi na costa próximo à província de Miyagi, a 373Km de Tóquio. A energia liberada pelo terremoto produziu um enorme tsunami. Uma hora após o terremoto, casas e campos em cidades localizadas na costa marítima foram destruídas 
por massivas e sucessivas ondas de 4 a 5 metros de altura (Figura 1).

De acordo com o portal de notícias Portalmie (2017), o Tsunami causado pelo terremoto ocasionou um desastre nuclear, pois atingiu a usina Fukushima Daiichi, causou uma falha no sistema de refrigeração e, assim, um colapso de reator nuclear com posterior liberação de matérias radioativos (Figura 2).

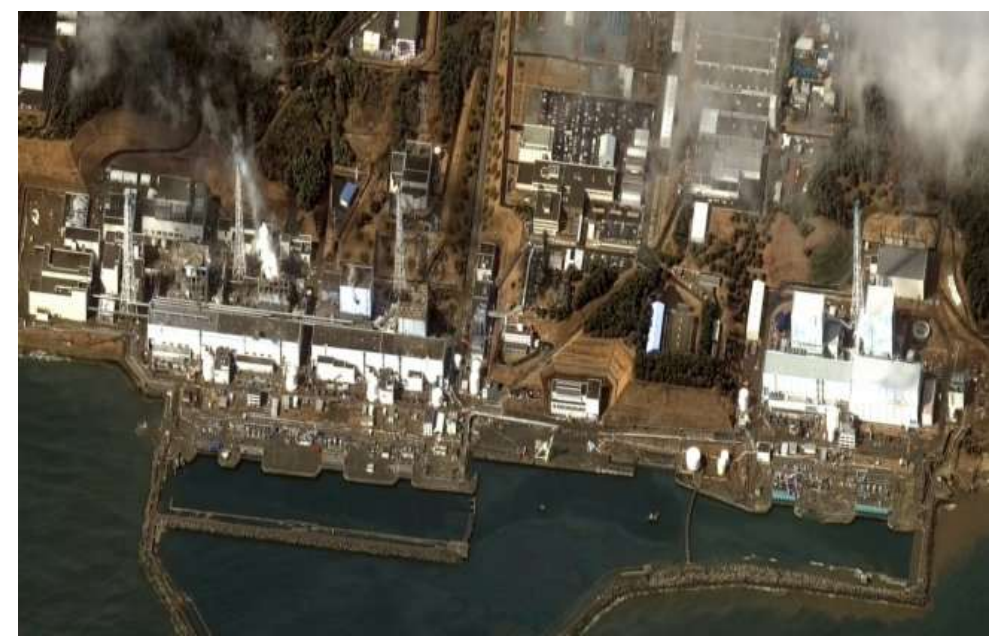

Figura 2: Imagem orbital da planta nuclear de Fukushima Daiichi, Japão. Fonte: AFP

Os materiais utilizados foram os dados coletados na página oficial do IERS (International Earth Rotation and Reference Systems Service). Para organizar e processar os dados, utilizou-se os softwares Excel 2016 e Gnuplot 4.4. Foram coletados junto ao IERS os dados de orientação da Terra, que são encontrados nos Boletins B, enumerados e coletados no intervalo do boletim B 278 até boletim B 285, que representam o período de setembro de 2010 a setembro de 2011. Com o uso do Excel 2016, tabelou-se e organizaram-se os dados, os quais se referem ao movimento do polo de $\mathrm{x}$ e y na unidade de milésimo de segundo (mas). Feito a organização, houve a criação do gráfico no próprio software. Determinaram-se também as coordenadas polares, calculadas por fórmula seguinte.

$$
\theta=\operatorname{arcos}\left(\frac{x}{d}\right)
$$

Onde x é a coordenada do polo do eixo x (em milésimo de segundo) e d é a distância polar, dada pela equação seguinte. Sendo x e y as coordenadas polares em milésimo de segundo.

$$
d=\sqrt{x^{2}+y^{2}}
$$

\section{RESULTADOS E DISCUSSÕES}

Conforme explicado em tópicos anteriores, o período escolhido refere-se a um intervalo de um pouco mais de seis meses antes e depois (totalizando um pouco mais de um ano) do terremoto que atingiu o Japão em 2011. Alves et al. (2008), concluíram que o resultado encontrado era o esperado: um movimento do polo de forma suave e cíclica. 


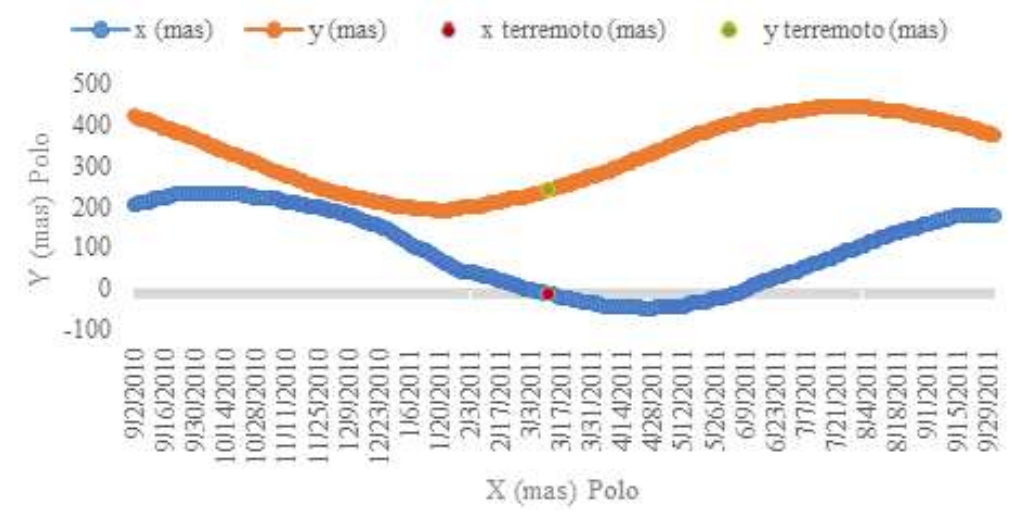

Figura 3: Coordenadas Xp e Yp do polo terrestre.

Apesar dos dados referentes às coordenadas instantâneas do polo terrestre em relação ao polo fixo da Terra, no trabalho supra estarem baseados em um período maior, a relação entre aquele e este trabalho ficam evidentes, uma vez que, vide Figura 3, o movimento é cíclico e sem alterações bruscas. As coordenadas polares, D e Teta $(\theta)$, calculadas por meio da fórmula S são apresentadas na Figura 4 a seguir.

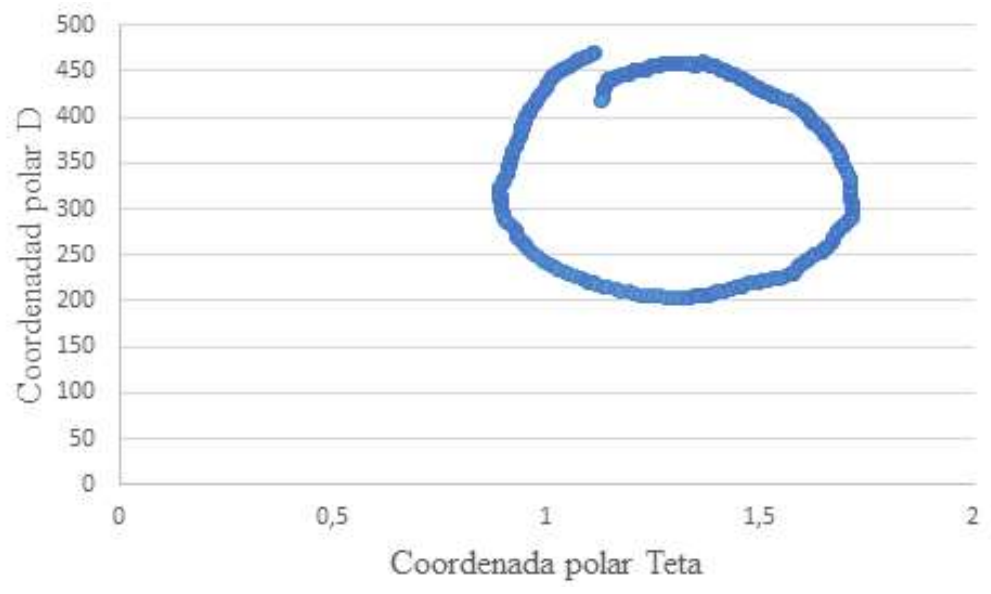

Figura 4: Coordenadas polares D e Teta.

Observa-se, na figura X, o quase círculo que caracteriza o Movimento de Chandler, o qual é cíclico (aparência circular). Novamente, este é um resultado coincidente ao de Alves, Souza e Monico (2008), uma vez que o movimento em questão é predito e sustentado por formulações matemáticas, as quais garantem sua verificação. A fim de facilitar a visulização, pois a projeção bidimensional pode ocasionar uma interpretação errônea do movimento, definiu-se um gráfico tridimensional (Figura 5) no qual se podem observar as coordenadas XPolo, YPolo (ambas em milisegundos) e sua oscilação ao longo tempo.

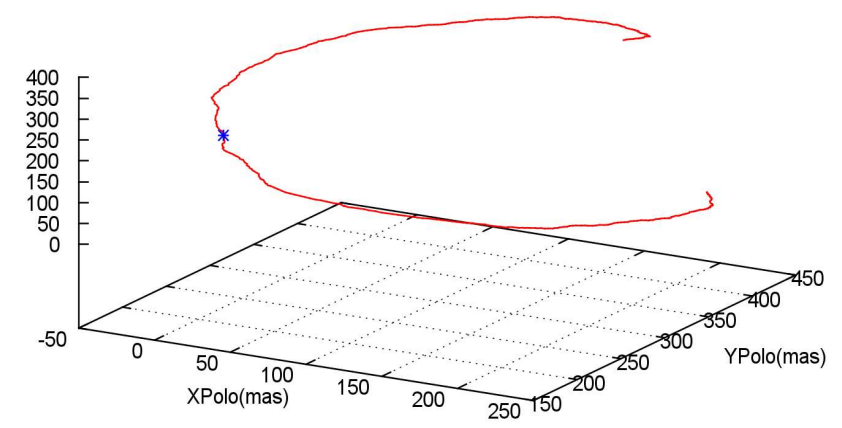

Figura 5: Movimento do polo em função do tempo (em dias). 


\section{CONCLUSÕES}

Este relatório teve por objetivo analisar o Movimento do Polo antes, durante e depois do terremoto de 2011, no Japão, o qual foi atingido. As figuras expostas no tópico anterior demonstram que as oscilações obedeceram ao padrão cíclico do Movimento de Chandler, de modo que é possível inferir que, apesar da magnitude do evento, não houve interferência sobre o movimento analisado.

Ressalta-se que esta atividade inspira a busca por novos conhecimentos e o aprofundamento de temas uma vez estudados, de modo que contribui sobremaneira para a formação acadêmica do discente, além de agregar valor e know hall sobre distintos assuntos.

\section{REFERÊNCIAS}

ALVES, D. B. M.; SOUZA E. M.; MONICO, J. F. G.. Movimento do Polo Terrestre: aspectos físicos, modelagem matemática e análises. Tend. Mat. Apl. Comput., v.9, n.1, p.1-10, 2008.

BBC. Terremoto no Japão é $7^{\circ}$ mais forte da história, 2011.
MIE. Março de 2011: Japão marca 6 anos do grande terremoto de Tohoku, 2017

SANTIAGO, B.; SALVIANO, A.. Astronomia geodésica posicionamento pelas estrelas, 2016.

A CBPC - Companhia Brasileira de Produção Científica (CNPJ: 11.221.422/0001-03) detém os direitos materiais desta publicação. Os direitos referem-se à publicação do trabalho em qualquer parte do mundo, incluindo os direitos às renovações, expansões e disseminações da contribuição, bem como outros direitos subsidiários. Todos os trabalhos publicados eletronicamente poderão posteriormente ser publicados em coletâneas impressas sob coordenação da Sustenere Publishing, da Companhia Brasileira de Produção Científica e seus parceiros autorizados. Os (as) autores (as) preservam os direitos autorais, mas não têm permissão para a publicação da contribuição em outro meio, impresso ou digital, em português ou em tradução. 